\title{
Coherence Singularities: Birth, Evolution, Possible Applications
}

\author{
(Invited Paper) \\ Tatiana Alieva ${ }^{1 *}$, Eugeny Abramochkin ${ }^{2}$ and José A. Rodrigo ${ }^{1}$ \\ ${ }^{1}$ Universidad Complutense de Madrid, Facultad de Ciencias Físicas, Ciudad Universitaria s/n, Madrid 28040, Spain \\ ${ }^{2}$ Lebedev Physical Institute, Samara, Russia \\ Email: talieva@ucm.es
}

\begin{abstract}
Coherent singular beams, in particular optical vortices, are attractive for different applications: free-space optical communication, imaging, particle manipulation, etc. However, their deformation during propagation through random media and speckle noise forced to look to their partially coherent analogues. The singularities of partially coherent beams associated with zero points of the cross-correlation function have been theoretically predicted and experimentally demonstrated for some particular cases of Laguerre-Gaussian (LG) and HermiteGaussian (HG) Schell-model beams (SMBs). Here we establish a theoretical background for explanation of these singularities evolution during propagation of the SMBs associated with structurally stable Hermite-Laguerre-Gaussian modes, which include as a particular case the LG and HG ones. The derived a closedform expression for the evolution of the mutual intensity of such beams allows easily calculating the intensity distribution and cross-correlation function at every plane of paraxial optical system. The birth and evolution of the cross-correlation singularities is analyzed. Their structure in far field serves as a fingerprint of the associated mode while the intensity distribution may not resemble the mode shape. The robustness of these singularities can be exploited for information encoding and random medium monitoring.
\end{abstract}

\section{INTRODUCTION}

Coherent structurally stable optical beams, in particular Gaussian optical vortices, which propagate without changing their form (apart from scaling and additional quadratic phase) are nowadays applied in different areas: free-space optical communication, imaging, particle manipulation, etc. Their easily recognized intensity patterns with typical for every beam zero lines associated with phase singularities make them attractive for information encoding. The Hermite-LaguerreGaussian (HLG) modes which include Hermite-Gaussian (HG), Laguerre-Gaussian (LG) ones are the mostly used beams for this purpose. However, their deformation during propagation through random media and speckle noise forced to look to their partially coherent analogues. Scalar paraxial partially coherent beams are described by mutual intensity (MI) defined as $\Gamma\left(\mathbf{r}_{1}, \mathbf{r}_{2}\right)=\left\langle f\left(\mathbf{r}_{1}\right) f^{*}\left(\mathbf{r}_{2}\right)\right\rangle$ [1] where $f(\cdot)$ is a complex field amplitude, $\mathbf{r}=(x, y)^{t}$ is a position vector at the plane transverse to the beam propagation direction $z$ and the brackets $\langle\cdot\rangle$ stand for ensemble averaging. In the case of Gaussian Schell model beams (SMBs) [2] the MI in the input plane is written as $\Gamma\left(\mathbf{R}+\frac{\mathbf{r}}{2}, \mathbf{R}-\frac{\mathbf{r}}{\mathbf{2}}\right)=f_{c}\left(\mathbf{R}+\frac{\mathbf{r}}{2}\right) f_{c}^{*}\left(\mathbf{R}-\frac{\mathbf{r}}{2}\right) \gamma(\mathbf{r})$, where the coordinates $\mathbf{R}=\frac{1}{2}\left(\mathbf{r}_{1}+\mathbf{r}_{2}\right)$ and $\mathbf{r}=\mathbf{r}_{1}-\mathbf{r}_{2}$ have been introduced, $\gamma(\mathbf{r})=\exp \left[-\frac{1}{2} \pi \sigma|\mathbf{r}|^{2}\right]$ is a degree of spatial coherence (DoC) which has a typical Gaussian form, and $f_{c}(\bullet)$ is the complex field amplitude of an associated coherent beam (HLG mode in our case). Thus, for $\mathbf{r}=0$ the MI corresponds to the intensity distribution $I(\mathbf{R})=$ $\Gamma(\mathbf{R}, \mathbf{R})=\left|f_{c}(\mathbf{R})\right|^{2}$, while for $\mathbf{R}=0$ the cross-correlation function $(\mathrm{CCF})$ used for the coherence singularities analysis is obtained $C(\mathbf{r})=\Gamma(\mathbf{r},-\mathbf{r})=f_{c}(\mathbf{r}) f_{c}^{*}(-\mathbf{r}) \gamma(2 \mathbf{r})$. The HLG modes considered here are defined as [3]

$$
\mathrm{HLG}_{n, m}(\mathbf{r} \mid \beta)=\sqrt{\frac{2}{2^{n+m} n ! m !}} \exp \left(-\pi|\mathbf{r}|^{2}\right) H L_{n, m}(\mathbf{r} \mid \beta) \text {, }
$$

where

$$
\begin{gathered}
H L_{n, m}(x, y \mid \beta)=(-i)^{m}(\cos 2 \beta)^{\frac{n+m}{2}} \\
\times \sum_{k=0}^{\min (n, m)}(-2 i \tan 2 \beta)^{k} k !\left(\begin{array}{c}
m \\
k
\end{array}\right)\left(\begin{array}{l}
n \\
k
\end{array}\right) \\
\times H_{n-k}\left(\sqrt{2 \pi} \frac{x \cos \beta+i y \sin \beta}{\sqrt{\cos 2 \beta}}\right) \\
\times H_{m-k}\left(\sqrt{2 \pi} \frac{y \cos \beta+i x \sin \beta}{\sqrt{\cos 2 \beta}}\right) .
\end{gathered}
$$

In particular, for $\beta=0$ and $\beta=\pi / 4$ they are reduced to the $\mathrm{HG}$ and the $\mathrm{LG}$ modes correspondingly. Taken into account the symmetry of the HLG modes, $H L_{n, m}(-\mathbf{r} \mid \beta)=$ $(-1)^{n+m} H L_{n, m}(\mathbf{r} \mid \beta)$, it is easy to see that in the input plane the coherence singularities (zero points of the $\mathrm{CCF}$ ) coincide with zero intensity points of the HLG mode since $C(\mathbf{r})=$ $(-1)^{n+m}\left|\mathrm{HLG}_{n, m}(\mathbf{r} \mid \beta)\right|^{2} \gamma(2 \mathbf{r})$. These simple expressions for the MI, the intensity and the CCF hold, however, only in the input plane and are destroyed during beam propagation.

In general, the spatial correlation singularities of partially coherent beams in contrast to the coherent ones can exist in the points where the intensity is not vanished [4]. In particular, it has been found that in far field the CCF phase singularity curves for the LG-SMBs have circular form [5], [6] and the number of such rings is empirically predicted to be $2 p+|l|$ [7], where $p=\min (n, m)$ and $l=n-m$ are the radial and azimuthal mode indices of the associated LG mode. It has been also shown that the HG-SMBs have linelike CCF singularities and that their number increases during beam propagation [8]. However, a theoretical background for 


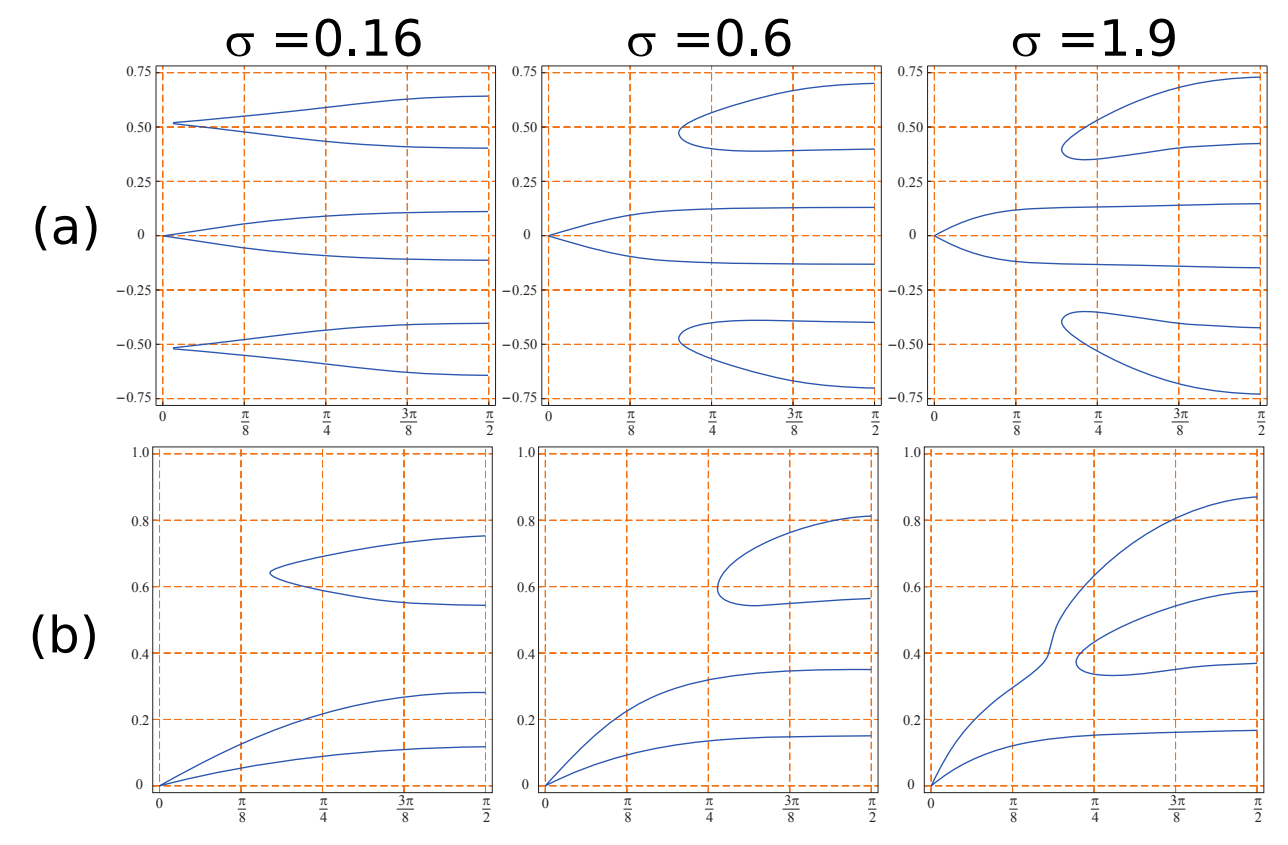

Figure 1. Evolution of the zero points of the CCF for fixed value of $x=0$ during propagation ( $y \alpha$-planes, where $\alpha$ is a horizontal coordinate and $y$ is a vertical one) for (a) HG-SMB $\operatorname{HLG}_{0,3}(\mathbf{r} \mid 0)$ and (b) LG-SMB HLG ${ }_{3,1}(\mathbf{r} \mid \pi / 4)$ for different parameter $\sigma$. The input coherence length corresponds to $w / \sqrt{\sigma}$, with $w$ being the fundamental Gaussian beam waist. Only positive $y$ coordinate is represented in b) taken into account the rotational symmetry of the LG-SMB.

explanation of singularity evolution during beam propagation have not been established. Here, we present a closed-form expression for the evolution of the mutual intensity of the HLG-SMBs which allows analyzing the birth and evolution of the coherence singularities during beam propagation.

\section{EVOLUTION OF THE CROSS-CORRELATION FUNCTION OF THE HLG-SMBS}

In order to find the expression for the MI and therefore the intensity and the CCF evolution during the HLG-SMBs propagation we as well as in [8] first express the MI through the ambiguity function (AF) which is presented as a product of the AF of the HLG mode $A_{\mathrm{HLG}_{n, m}(\bullet \mid \beta)}$ and the DoC. Note, that the beam propagation in rotationally symmetric system is primary described by the symmetric fractional Fourier transform (FT) and the HLG modes are its eigenfunctions [9]. Then the $A_{\mathrm{HLG}_{n, m}(\bullet \mid \beta)}$ does not change during propagation and the expression for the evolution of the MI can be found from

$$
\begin{aligned}
& \Gamma_{\mathrm{HLG}_{n, m}(\bullet \mid \beta)}\left(\mathbf{R}+\frac{\mathbf{r}}{2}, \mathbf{R}-\frac{\mathbf{r}}{2} \mid \alpha\right)=\int A_{\mathrm{HLG}_{n, m}(\bullet \mid \beta)}(\mathbf{r}, \mathbf{p}) \\
& \quad \times \exp \left(-\frac{\pi}{2} \sigma|\mathbf{r} \cos \alpha-\mathbf{p} \sin \alpha|^{2}\right) \exp \left(i 2 \pi \mathbf{R}^{\mathbf{t}} \mathbf{p}\right) \mathrm{d} \mathbf{p}, \quad \text { (3) }
\end{aligned}
$$

where $\alpha$ is a parameter of the fractional FT (related to the propagation distance as $z=\tan \alpha, \alpha \in[0, \pi / 2])$. Here dimensionless coordinates are used for simplicity. Finding the expression for the $A_{\mathrm{HLG}_{n, m}(\bullet \mid \beta}$ and after long but straightforward calculus, which can be found in [10], we derive the expression for the MI of the HLG-SMB

$$
\begin{gathered}
\Gamma_{\mathrm{HLG}_{n, m}(\bullet \mid \beta)}\left(\mathbf{R}+\frac{\mathbf{r}}{2}, \mathbf{R}-\frac{\mathbf{r}}{2} \mid \alpha\right)=\frac{1}{\left(1+\sigma \sin ^{2} \alpha\right)^{n+m+1}} \\
\times \exp \left(-\frac{2 \pi|\mathbf{R}|^{2}-i \pi \sigma \mathbf{R}^{\mathrm{t}} \mathbf{r} \sin 2 \alpha+\frac{1}{2} \pi|\mathbf{r}|^{2}(1+\sigma)}{1+\sigma \sin ^{2} \alpha}\right) \\
\times \sum_{\nu=0}^{n} \sum_{\mu=0}^{m}\left(\begin{array}{c}
n \\
\nu
\end{array}\right)\left(\begin{array}{c}
m \\
\mu
\end{array}\right) \frac{\left(\sigma \sin ^{2} \alpha\right)^{n+m-\nu-\mu}}{2^{\nu+\mu-1} \nu ! \mu !} \times \\
H L_{\nu, \mu}\left(\frac{\mathbf{R}+\frac{1}{2} q(\alpha, \sigma) \mathbf{r}}{\sqrt{1+\sigma \sin ^{2} \alpha}} \mid \beta\right) H L_{\nu, \mu}^{*}\left(\frac{\mathbf{R}-\frac{1}{2} q(\alpha, \sigma) \mathbf{r}}{\sqrt{1+\sigma \sin ^{2} \alpha}} \mid \beta\right),
\end{gathered}
$$

where $q(\alpha, \sigma)=1+\sigma \sin ^{2} \alpha-i \sigma \sin \alpha \cos \alpha$. We observe that the intensity distribution $I_{\mathrm{HLG}_{n, m}(\bullet \mid \beta)}(\mathbf{R} \mid \alpha)$ obtained from Eq. (4) for $\mathbf{r}=\mathbf{0}$ is expressed as a superposition of the intensity distributions of the HLG modes of equal or lower indices and does not have zeros (note, that the roots of the HL polynomials never coincide) except of the input plane corresponding to $\alpha=0$ where only one mode is present in the sum. On the other hand, the Eq. (4) for $\mathbf{R}=\mathbf{0}$ is reduced to the $\mathrm{CCF}$ :

$$
\begin{gathered}
C_{\mathrm{HLG}_{n, m}(\bullet \mid \beta)}(\mathbf{r} \mid \alpha)=\frac{1}{\left(1+\sigma \sin ^{2} \alpha\right)^{n+m+1}} \\
\times \exp \left(-\frac{2 \pi|\mathbf{r}|^{2}(1+\sigma)}{1+\sigma \sin ^{2} \alpha}\right) \sum_{\nu=0}^{n} \sum_{\mu=0}^{m}(-1)^{\nu+\mu}\left(\begin{array}{l}
n \\
\nu
\end{array}\right)\left(\begin{array}{c}
m \\
\mu
\end{array}\right) \\
\times \frac{\left(\sigma \sin ^{2} \alpha\right)^{n+m-\nu-\mu}}{2^{\nu+\mu-1} \nu ! \mu !}\left|H L_{\nu, \mu}\left(\frac{q(\alpha, \sigma) \mathbf{r}}{\sqrt{1+\sigma \sin ^{2} \alpha}} \mid \beta\right)\right|^{2} .
\end{gathered}
$$


Here the polynomial symmetry has been used again. The analysis of the CCF singularities is related to search of the real roots of the $2 \mathrm{D}$ polynomial of order $2(n+m)$ written as a sum in this equation. Even for the same HLG mode the form of the root (and therefore singularity) curves changes depending on the parameters $\alpha$ and $\sigma$. From the symmetry of the HG and LG modes it follows that singularity curves are lines and rings correspondingly. In Fig.1a and Fig.1b the evolution of zero points of CCF for fixed value of $x=0$ during propagation ( $y \alpha$-plane) is displayed for HG-SMB (a) and LG-SMB (b), correspondingly. It can be seen that the singularity points appear and change their position during beam propagation. While in far field, $\alpha=\pi / 2$, the number and position of the singularity points are almost independent of the coherence parameter $\sigma$ they are significantly vary during propagation. The singularity lines of the more complex forms are observed for general HLG-SMB [11].

\section{DISCUSSION}

The derived equation for the MI evolution during HLGSMB propagation through rotationally symmetric systems apart from its fundamental value is useful for beam analysis. Indeed, it allows predicting the intensity distortion during propagation and demonstrating that every HLG-SMB has its own fingerprint recorded in the form and number of the CCF singularity curves in far field. Taken into account the robustness of the partially coherent beam during propagation through random medium it might be interesting to evaluate the possibility of information encoding in CCF singularity curves rather than in phase singularities of the coherent modes. On the other side if the form of the CCF singularities is significantly alternated by the random medium then the analysis of these can be used for medium monitoring.

\section{ACKNOWLEDGMENT}

The Spanish Ministerio de Economía y Competitividad is acknowledged for the project TEC2014-57394-P.

\section{REFERENCES}

[1] M. Born and E. Wolf, Principles of Optics. Cambridge University Press, UK, 2006

[2] A. C. Schell, "The multiple plate antenna," Ph.D. dissertation, Massachusetts Institute of Technology, 1961.

[3] E. G. Abramochkin and V. G. Volostnikov, "Generalized Gaussian beams," Journal of Optics A: Pure and Applied Optics, vol. 6, no. 5, pp. S157-S161, 2004

[4] F. Gori et al, "Partially coherent sources with helicoidal modes," J. Mod. Opt., vol. 45, no. 3, pp. 539-554, 1998.

[5] I. D. Maleev et al, "Spatial correlation vortices in partially coherent light: theory," J. Opt. Soc. Am. B, vol. 21, no. 11, pp. 1895-1900, 2004

[6] D. M. Palacios et al, "Spatial correlation singularity of a vortex field," Phys. Rev. Lett., vol. 92, no. 14, ID 143905, 2004.

[7] Y. Yang et al, "Effect of the radial and azimuthal mode indices of a partially coherent vortex field upon a spatial correlation singularity," New J. Phys., vol. 15, no. 11, ID 113053, 2013.

[8] J. A. Rodrigo and T. Alieva, "Evolution of coherence singularities of Schell-model beams," Optics Letters, vol. 40, no. 15, pp. 3635-3638, 2015.

[9] E. Abramochkin et al, Solutions of paraxial equations and families of Gaussian beams in Mathematical Optics: Classical, Quantum, and Computational Methods, V. Lakshminarayanan, M. L. Calvo and T. Alieva Eds., CRC Press, New York, USA, pp. 143-192, 2013.
[10] E. Abramochkin and T. Alieva, "Closed-form expression for mutual intensity evolution of Hermite-Laguerre-Gaussian Schell-model beams," Optics Letters, vol. 42, accepted for publication, 2017.

[11] T. Alieva et al, "Evolution of coherence singularities of beams associated with structurally stable Gaussian modes,' in Proceedings Frontiers in Optics 2016, Rochester, New York, USA, FF5G.4, 2016. 